\title{
MODELO PARA ESTIMACIÓN DE ÁREA FOLIAR EN BERENJENA (Solanum melongena L) BASADO EN MUESTREO NO DESTRUCTIVO
}

\section{A NON-DESTRUCTIVE SAMPLING MODEL FOR ESTIMATING LEAF AREA IN EGG PLANT (Solanum melongena L)}

\author{
Carlos E. Cardona ${ }^{1}$, Hermes Araméndiz ${ }^{1}$, Carlos J. Barrera ${ }^{2}$
}

\begin{abstract}
RESUMEN
La investigación se realizó en Montería, a 13 m.s.n.m., temperatura media de $27,4{ }^{\circ} \mathrm{C}$, precipitación anual de 1346,1 mm, humedad relativa del $84 \%$ y brillo solar anual de 2180,2 horas, con el fin de obtener un modelo que permitiera estimar el área foliar a partir de muestreo no destructivo. Mediante muestreo aleatorio simple, se seleccionaron hojas sanas de plantas adultas de diferentes tamaños y distintas etapas de desarrollo de los doseles superior, medio e inferior de la variedad de berenjena Criollo lila. Se tomaron medidas longitudinales (largo de la hoja, ancho de la misma a 1/5, 1/3, 1/2 y 2/3 de su longitud) para determinar las medidas altamente relacionadas con el área fotosintética de la hoja. El área foliar (Y) se midió por el método de relación peso (área sobre una muestra de 30 hojas) por dosel. Se construyeron modelos de regresión simple y múltiple para luego seleccionar el de mejor ajuste. Se seleccionó el modelo de regresión simple cuya variable explicativa corresponde al largo de la hoja $(X)$. El modelo de mejor ajuste fue $\hat{Y}=-49,10+8,33 X, R^{2}=0,9605$
\end{abstract}

Palabras clave: Solanum melongena, hojas, área foliar, modelo de regresión.

\begin{abstract}
This research was done in Montería at 13 m.a.s.l., average temperature $27,4{ }^{\circ} \mathrm{C}$, $1346.1 \mathrm{~mm}$ annual rainfall, $84 \%$ relative humidity and 2180,2 daily light hours annually to obtain a model to estimate plant leaf area based on a non-destructive sampling. A probability sampling procedure was used. Leaves of different sizes and stages of development were selected from the upper, middle and lower canopy of adult plants. Leaf length and width were taken at 1/5, 1/3, 1/2 y 2/3 of the leaves to identify highly related photosynthetic leaf area measures. The leaf area $(\mathrm{Y})$ was measured using the weight (30 leaves sample area) per canopy. Simple linear regression models and multiple linear regression were adjusted. The simple linear regression model whose independent variable was the length leaf $(X)$ was selected. The model was $\hat{Y}=-49,10+8,33 X, R^{2}=0,9605$
\end{abstract} Keywords: Solanum melongena, leaves, leaf area, regression model.

\footnotetext{
${ }^{1}$ Universidad de Córdoba, Departamento de Ingeniería Agronómica y Desarrollo Rural, Carrera 6 No 76 - 103, Montería- Córdoba. Telefax (4) 786 0255. E-mail: ccardonaayala@yahoo.co

${ }^{2}$ Instituto Tecnológico Metropolitano, Calle 54 No 30-01, Barrio Boston, Medellín-Antioquia. E-mail: carlosbarrera@itm.edu.co
} 


\section{INTRODUCCIÓN}

El departamento de Córdoba es el principal productor de berenjena en Colombia con 220 ha en áreas que fluctúan entre 1.000 y $2.500 \mathrm{~m}^{2}$. Esta especie vegetal es una hortaliza de gran importancia en el sector de economía campesina en el Caribe Húmedo, arraigada a la cultura culinaria como legado de la migración árabe al comienzo del siglo XX (Aramendiz et al. 2008).

A nivel mundial, los sectores hortofrutícola y de tubérculos han crecido a tasas de 4,5 y 4,3\%, respectivamente; en tanto que a nivel nacional se registra un rezago tecnológico y se han perdido oportunidades externas conquistadas por otros países como México y Honduras, que contribuyen a la satisfacción de la mayor demanda de los Estados Unidos y Canadá que se registra entre los meses de noviembre y marzo (Cardona et al. 2008). En el caso de la berenjena, cultivo de economía campesina y de pequeñas aéreas de siembra, el rezago tecnológico se fundamenta, entre otros aspectos, en el desconocimiento de muchos tópicos de la eficiencia fisiológica de la planta, entre los cuales está la medida de la asimilación de carbono llevada a cabo por las hojas durante el proceso fotosintético, que influye en el manejo que se le podría dar al sistema de producción con fines de alto rendimiento y competitividad.

Las hojas constituyen el órgano más importante de la planta y juegan el papel principal en las actividades anabólicas por medio de la clorofila que poseen en abundancia, el único medio para los procesos fotosintéticos. El área foliar total, que ha sido directamente relacionada con la cantidad de clorofila, es un parámetro 
importante para estimar la habilidad de la planta para sintetizar materia seca. Su adecuada determinación durante el ciclo del cultivo posibilita conocer el crecimiento y el desarrollo de la planta, la eficiencia fotosintética y, en consecuencia, la producción total de la planta (Teruel 1995, Costa 1999, Campostrini y Yamanishi 2001). Adicionalmente, ayuda en la definición de la época ideal de siembra y de trasplante; si no se tienen en cuenta otros factores, los cultivos deben ser sembrados en épocas en las cuales el máximo valor de índice del área foliar coincida con la época de elevada radiación, cuando la fotosíntesis líquida sea máxima, y también contribuye a estimar las necesidades hídricas de los cultivos, por lo que se requieren modelos matemáticos sencillos y rápidos para su estimación (Allen et al. 1997, Galvani et al. 2000, Guo y Sun 2001, Arjona 2003).

Los métodos directos de medición de área foliar están restringidos al uso de equipos automáticos costosos; el empleo de planímetros resulta muy tedioso y en algunos experimentos el tiempo es insuficiente para realizar tales mediciones. Sin embargo, la estimación del área foliar a través de modelos matemáticos con mediciones directas en las hojas es bastante preciso, económico y no destructivo (Manivel y Weaver 1974, Blanco y Folegatti 2003). Recientes estudios y modelaciones de las interacciones entre los regímenes dinámicos de radiación y el desarrollo de las hojas, en conjunto, se han enfocado sobre la interceptación total de la energía radiante como un factor predictor de la producción de materia seca (Campostrini y Yamanishi 2001). 
Un modelo matemático es una simplificación del sistema real y consiste en una ecuación o juego de ecuaciones, las cuales representan cuantitativamente las hipótesis acerca del sistema real. Estos modelos se emplean en muchos campos y a través de ellos se han hecho simulaciones y se han elaborado ecuaciones para facilitar las mediciones en el crecimiento de las plantas cultivadas (Thornely 1976, Bidwel 1984, Elser y Jubb 1988, Kingston y Van Epenhuijsen 1989, Montoya et al. 1991). El desarrollo de modelos matemáticos a partir de medidas lineales de una lámina foliar para predecir su área fotosintética se ha usado en diversos estudios de crecimiento y desarrollo de diferentes especies, siendo algunas de las medidas más comunes tenidas en cuenta en las ecuaciones de estimación la longitud de la hoja, el ancho de la hoja, la longitud del pecíolo, el diámetro del pecíolo, o combinaciones entre estas variables (Montoya et al. 1991, Gamiely et al. 1991, Cardona et al. 2009).

Los métodos no destructivos se fundamentan en mediciones lineales que son rápidas, fáciles de medir y permiten una buena precisión en estudios de crecimiento de muchas especies vegetales, donde el modelo matemático puede obtenerse por correlación entre la longitud de la hoja (L), ancho (W) o longitud por ancho (LW) de área foliar (LA) de una muestra de hojas a través del análisis de regresión (Manivel y Weaver 1974, Sepaskhah 1977, Chirinos et al. 1997, Guo y Sun 2001, Arjona 2003, Blanco y Folegatti 2003, Espitia et al. 2006, Cardona et al. 2009, Cabezas-Gutiérrez et al. 2009). 
Ante la importancia de la berenjena como hortaliza posicionada en el mercado nacional y con perspectivas muy favorables para exportación, se hace necesario contribuir al desarrollo del cultivo como sistema de producción eficiente, ya que el conocimiento de la cinética del crecimiento y desarrollo de la planta permite su manejo agronómico en aspectos asociados al manejo de las necesidades hídricas de la planta, programación de planes de fertilización para reducir costos y ser mucho más competitivos en la producción. Por lo tanto, el objetivo del presente trabajo fue obtener un modelo para estimar el área foliar con la toma de medidas directas de las hojas en campo, sin utilizar el muestreo destructivo.

\section{MATERIALES Y METODOS}

La investigación se realizó en condiciones de campo y de laboratorio en la Universidad de Córdoba (Montería-Colombia), ubicada a una altura de 13 m.s.n.m., con coordenadas geográficas de $8^{\circ} 48^{\prime}$ de latitud Norte y $75^{\circ} 52^{\prime}$ de longitud Oeste, en un bosque seco tropical, según la clasificación de Holdrige, con precipitación anual de $1.346,1 \mathrm{~mm}$, temperatura media del aire de $27,4{ }^{\circ} \mathrm{C}$, humedad relativa de 84\% y brillo solar anual de 2.108,2 horas (Palencia et al. 2006).

Se realizó un muestreo aleatorio simple para la selección de hojas. Se tomaron diferentes tamaños en diferentes etapas de desarrollo de las plantas de un cultivo establecido con la variedad “Criollo lila”, sembrada a $1 \mathrm{~m}$ entre plantas y $1 \mathrm{~m}$ entre hileras, a partir de los 15 días después del trasplante. La determinación de las 
variables o medidas de las hojas relacionadas con el área foliar, se hizo sobre una muestra de 30 hojas de cada uno de los doseles (superior, medio e inferior) de plantas adultas determinados por altura, y seleccionando hojas sanas de distintos tamaños. Las mediciones del largo y ancho de la hoja, se realizaron con una regla milimetrada a 1/5, 1/3, 1/2 y 2/3 de su longitud, como variable dependiente el área Foliar (AF). El área foliar, se obtuvo por el método de relación peso: área (o del sacabocado). Los discos de lámina foliar obtenidos con un sacabocados de 2,3 cm de diámetro, midieron 4,1548 $\mathrm{cm}^{2}$. A cada hoja de la muestra se le determinó su masa fresca extrayendo de cuatro a seis discos de tejidos de cada lámina foliar. Posteriormente, se obtuvo la masa fresca de cada uno y se halló la media y el coeficiente de variación de los discos (Pire y Valenzuela 1995). Seguidamente, se calculó el área foliar de cada hoja a través de la relación entre el peso fresco y el área unitaria del disco así:

$$
\text { Área foliar calculada }=\frac{\text { Peso totalde la hoja } x \text { Área del disco }}{\text { Peso promediode los discos }}
$$

El análisis de las relaciones entre las medidas de las hojas y el área foliar se hizo mediante correlación simple y múltiple, con el fin de extraer información sobre el grado de asociación lineal entre las variables área foliar y las medidas lineales de las hojas. Se aplicó el paquete estadístico SAS versión 9.1 (SAS 2007) para la construcción y la selección de los modelos mediante la técnica "STEPWISE" (regresión paso a paso), la cual tiene como criterio de selección el $\mathrm{R}^{2}$ máximo y una prueba de significancia F o un estadístico Cp. Este método consiste en construir, de manera iterativa, una secuencia de modelos de regresión mediante la adición o 
eliminación de variables en cada paso. El criterio para añadir o eliminar una variable en cualquier paso se expresa de manera fácil en términos de una prueba parcial F. Este tipo de regresión por pasos es una versión modificada del proceso de regresión hacia delante, en la que en cada nuevo paso cuando se incluye una nueva variable, además se reconsidera el mantener las que ya se habían añadido previamente. Es decir, que no sólo puede entrar una nueva variable en cada paso sino que puede salir alguna de las que ya estaban en la ecuación. El proceso finaliza cuando ninguna variable de las que no están en la ecuación cumple la condición para entrar y de las incorporadas a la ecuación ninguna cumple la condición para salir (Montgomery y Runger 1996, Rawlings et al. 2001)

El estadístico Cp es también una herramienta para la selección del modelo. Cp es definido como:

$C_{p}=p+\frac{\left(s^{2}-\hat{\sigma}^{2}\right)(n-p)}{\hat{\sigma}^{2}}$

Donde $\mathrm{p}$ es el número de parámetros en el modelo, $\mathrm{s}^{2}$ es el cuadrado medio del error para el modelo candidato, y $\hat{\sigma}^{2}$ una estimación de la varianza poblacional $\sigma^{2}$. Son deseables valores pequeños de Cp (Walpole, 1995).

Posteriormente se realizó una validación de los modelos, a partir de un nuevo conjunto de datos, en condiciones diferentes. Las pruebas más comunes sugeridas por la literatura estadística se emplearon para la validación de los supuestos del modelo: normalidad de los errores (prueba de Shapiro Wilk), heteroscedasticidad de los 
errores (prueba de White), independencia de los errores (prueba de Durbin Watson), aleatoriedad de los residuos (prueba de rachas o de secuencias) y diagnóstico de multicolinealidad, con la prueba de multicolinelidad, basada en la estructura la estructura de la matriz $X^{\prime} X$, que se puede escribir como $X^{\prime} X=V D^{2} V$, donde $V$ es una matriz ortogonal, cuyas columnas son los vectores propios de $X^{\prime} X, y D^{2}$, una matriz diagonal de valores propios asociados a los vectores propios correspondientes (Gujarati, 1997; Rawlings et al., 2001).

\section{RESULTADOS Y DISCUSIÓN}

\section{El modelo y la significancia de sus parámetros}

El análisis de regresión con el PROC REG y el proceso de selección de las variables predictoras mediante la técnica STEPWISE del programa estadístico SAS permitieron seleccionar como variables independientes, el largo de la hoja y el ancho de la misma a 2/3 de su largo, a partir del punto de inserción del pecíolo con la lámina foliar. La prueba $\mathrm{F}$ del análisis de varianza resultó altamente significativa $(\operatorname{Pr}>\mathrm{F}=0,0001)$, entonces el área foliar ( $\mathrm{AF}=\mathrm{Y}$ ) está relacionada linealmente con el largo de la hoja. (Figura 1; Tabla 1). Además, esta variable explica la variación del área foliar (AF=Y) en un $96,05 \%$, es decir $R^{2}=0,9605$.

El modelo de mejor ajuste resultó ser:

$\hat{Y}=-49,10+8,33 X, R^{2}=0,9605$ 
Según la ecuación estimada, con un error estándar de 0,32cm, por cada centímetro que se incremente el largo de la hoja ( $X$ ), el área foliar aumenta en $4,95 \mathrm{~cm}^{2}$, con efecto lineal (Figura 2, Tabla 2).

La línea de regresión estimada sólo es válida dentro del rango de valores de X, es decir, que no es conveniente extrapolar el uso de la línea para otros valores, pues la forma de la respuesta puede cambiar fuera de este rango (Gómez 1997). Así, los valores encontrados de medidas lineales del largo de la hoja de berenjena (X) variaron entre 7,8 y 25,7 cm, valores comunes en la especie según la etapa de desarrollo de la hoja.

Debe anotarse que $\widehat{\beta}_{0}$ intercepto cuyo valor en el modelo es de $-49,10 \mathrm{~cm}^{2}$ no tiene interpretación práctica, sólo es un término de ajuste que permite representar la tendencia de los datos (en el espacio de valores observados para la variable independiente) mediante un modelo de línea recta. Para que sea posible una interpretación de $\widehat{\beta}_{0}$ es necesario que sea físicamente posible que $\mathrm{X}$ tome el valor de cero y además, que se hayan recolectado datos con $\mathrm{X}=0$, lo cual es imposible cuando se trata de medir el área foliar (Gómez 1997, Díaz 1999, Melo et al. 2007).

Este resultado es similar al reportado por Nascimento et al. (2002) en pepino (Cucumis melo), quienes estimaron un modelo no lineal que relaciona el largo de la hoja con el área foliar total. Asimismo, Queiroga et al. (2003) encontraron un modelo potencial que permite estimar el área foliar del fríjol (Phaseolus vulgaris) por método 
no destructivo con la medida del largo máximo del folíolo central. Por otra parte, Pire y Valenzuela (1995) en vid (Vitis vinífera) encontraron modelos de regresión que utilizan el largo o el ancho de la hoja como variables explicativas para estimar el área foliar del cultivar "French Colombard" como alternativa cuando no se dispone de equipo electrónico integrador de área foliar; informan además que una mayor precisión se obtiene con modelos cuadráticos, siempre que se disponga de las dos medidas: largo y ancho, con modelos independientes. Contrastando con los resultados que utilizan modelos lineales basados en la medida del largo de la hoja, Rossini et al. (2004) encontraron en rosa mística (Zinnia elegans) y otras especies afines, modelos lineales con variables explicativas producto del largo por el ancho de la hoja.

Es claro que un modelo o ecuación que permite estimar el área foliar es de gran utilidad práctica, cuando se trata, por ejemplo, de evaluar el efecto de las distintas condiciones estresantes, de común ocurrencia en los sistemas de producción agrícolas. Tales condiciones, como las relacionadas con el estrés biológico, causado por artrópodos plaga y enfermedades criptogámicas, se manifiestan en disminuciones de área foliar por daño directo sobre las hojas o inducción de senescencia precoz de las mismas (Hidalgo et al. 2006). Es evidente, además, que bajo estrés hídrico los rendimientos también se reducen como consecuencia de la pérdida de área foliar (Ribas et al. 2000).nEs en estas situaciones que un modelo matemático es de gran utilidad, debido a que constituye una herramienta para dar respuesta a interrogantes, tales como: ¿qué tanto está disminuyendo o aumentando el área foliar total? y ¿cómo influye en los rendimientos?. En este orden de ideas, los ensayos de aplicación de 
pesticidas, fertilizantes, métodos de riego y otras prácticas agronómicas se pueden evaluar, con la ayuda de modelos matemáticos, utilizando los diseños experimentales o de muestreo apropiados (Cardona et al., 2009). Por otra parte, la estimación del área foliar, también es de gran utilidad cuando se trata de ajustar poblaciones de plantas para obtener el índice de área foliar crítico (IAF crítico) o sea, aquel que intercepta el 95\% de la radiación incidente con el consecuente aumento significativo de la tasa de crecimiento del cultivo (TCC). En la medida en que el IAF aumenta, la TCC también lo hace hasta que el dosel intercepta el 95\% de la radiación incidente; por encima de un IAF crítico, la TCC no incrementa significativamente (Monteith 1965, Squire 1990).

\section{Cumplimiento de los supuestos del modelo}

El modelo de mejor ajuste seleccionado cumplió con el supuesto del análisis de regresión, que establece que el error estándar de estimación es independiente para cada valor de la variable explicativa (Largo de la hoja), es decir, los errores no están correlacionados (prueba de Durbin-Watson). En esta misma dirección, la prueba de aleatoriedad (Rachas) mostró que los errores corresponden a un arreglo aleatorio, o bien no están autocorrelacionados, es decir son independientes, por lo que se determina que las muestras tomadas para la estimación del modelo son aleatorias y las sucesivas observaciones son independientes.

Para la prueba de normalidad, el estadístico de la prueba de normalidad de los errores de Shapiro-Wilk, obtenido por medio del PROC UNIVARIATE NORMAL del SAS, 
mostró que evidentemente los errores se distribuyen normalmente. Estadísticamente, según la prueba de White, no hay heterocedasticidad en los errores, por lo tanto la variación en torno a la recta de regresión es constante para todos los valores de $\mathrm{X}$ (Largo de la hoja), sin importar que los valores que tome sean altos o bajos, de todas formas, la variación se supone es la misma.

\section{CONCLUSIONES}

- El área foliar (AF) de berenjena está relacionada linealmente con el largo de la hoja, medido desde el punto de inserción del pecíolo.

- El modelo lineal encontrado para estimar el área foliar de berenjena “Criollo lila” puede ser usado en estudios agronómicos con rapidez y precisión

- El modelo lineal simple seleccionado y validado mostró mayor precisión para estimar el área foliar de berenjena y cumple con los supuestos de normalidad, homogeneidad de varianzas e independencia de los errores.

\section{REFERENCIAS}

Allen, R., Smith, M., Pereira, L. y Pruit, W 1997. Proposed revision to the FAO: Procedure for estimating crop water requeriments. Acta Horticulturae (ISHS) 449:17-34.

Araméndiz, H., Cardona, C., Jarma, A. y Espitia, M. 2008. El Cultivo de la Berenjena (Solanum melongena L). Editorial Produmedios, Bogotá, 152 p.

Arjona, H. 2003. Estimación del área foliar de cebolla de bulbo (Allium cepa L. Yellow Granex F1) mediante la aplicación de tres modelos estadísticos. Revista Comalfi 30(1):28-36. 
Bidwell, R. 1984. Fisiología Vegetal. Introducción y Generalidades. AGT Editorial, p286-315.

Blanco, F. y Folegatti, M. 2003. A new method for estimating the leaf area index of cucumber and tomato plants. Horticultura Brásileira 21(4):666-669.

Cabezas-Gutiérrez, M., Peña, F., Duarte, H., Colorado, F. y Silva, R. 2009. Un modelo para la estimación de área foliar en tres espécies forestales de forma no destructiva. Revista U.D.C.A. 12(1):121-131.

Campostrini, E. y Yamanishi, O. 2001. Estimativa da área foliar do mamoeiro utilizando o comprimento da nervadura central. Scientia Agrícola 58(1):39-42.

Cardona, C., Araméndiz, H. y Barrera, C. 2009. Estimación del área foliar de papaya (Carica papaya L) basada em muestreo no destructivo. Revista U.D.CA. 12(1):131-139

Cardona, C., Araméndiz, H. y Espitia, M. 2008. Biología floral y fisiologia reproductiva de la berenjena (Solanum melongena). Editorial Produmedios, Bogotá, p9-12.

Chirinos, D., Chirunos-Torres, J., Geraud-pouey,F., Fernández, R., Mármol, L. y Chirinos-Torres, D. 1997. Modelos para estimar el área foliar de melón híbrido Durango. Facultad de Agronomía. Revista LUZ 14:163-171.

Costa, M. 1999. Efeitos de diferentes lâminas de água com dois níveis de salinidade na cultura do meloeiro. Tesis Ph. D., Universidade Estatal de Sao Paulo, Botucatu, $115 p$.

Díaz, A. 1999. Diseño de Experimentos. Editorial Universidad de Antioquia, Medellín,347p.

Elser, E. y Jubb, G. 1988. Leaf area estimation of concord grape leaves from simple linear measurements. American Journal of Enology and Viticulture 39(1):95-97.

Espitia, M., Montoya, R., Robles, J., Barbosa, C. y Vergara, C. 2006. Modelo estadístico para estimación de área foliar em Stevia rebaudiana. Bertoni en el Sinú medio. Temas Agrarios 11(2):45-52.

Galvani, E., Eescobedo, J., Cunha, A. y Klosowski, E. 2000. Estimativa do índice de área foliar e da produtividade de pepino em meio protegido - cultivo de inverno e de verão. Revista Brasileira de Engenharia Agrícola e Ambiental 4:8-13.

Gamiely, S., Randel, W., Millas, H. y Smitle, D. 1991. A Rapid and nondestructive method for estimating leaf area of onions. HortScience 26(2):206.

Gómez, H. 1997. Estadística Experimental Aplicada a las Ciencias Agrícolas. Universidad Nacional de Colombia - Sede Medellín, Medellín, 571p.

Gujarati, D. 1997. Econometría. Editorial Mc Graw-Hill, Mexico D. F., p344-391. 
Guo, D. y Sun, Y. 2001. Estimation of leaf area of stem lettuce (Lactuca sativa var Angustana) from linear measurements. Indian Journal of Agricultural Sciences 71(7):483-486.

Hidalgo, M., Tapia, A., Rodríguez, W. y Serrano, E. 2006. Efecto de la sigatoka negra (Mycosphaerella fijiensis) sobre la fotosíntesis y transpiración foliar del banano Musa AAA cv. Valery. Agronomía Costarricense 30(1):35-41.

Kingston, C. y Van Epenhuijsen, C. 1989. Influence of leaf area on fruit development and quality of Italia glasshouse table grapes. American Journal of Enolology and Viticulture 40 (2):130-134.

Manivel, L. y Weaver, R. 1974. Biometric correlations between leaf area and length measurements of “Grenache” grape leaves. HortScience 9(1):27-28.

Melo, O., López, L. y Melo, S. 2007. Diseño de Experimentos Métodos y Aplicaciones. Pro-offset Editorial S.A., Bogotá, 668p.

Monteith, J. 1965. Light distribution and photosynthesis in field crops. Annals of Botany 29(113):17-37.

Montgomery, D. y Runger, G. 1996. Probabilidad y Estadística Aplicadas a la Ingeniería. McGraw-Hill Interamericana Editores, S.A., México D. F., 895p.

Montoya, R., Hernández, M. y Clavijo. J. 1991. Determinación de modelos matemáticos para medición de área fotosintética y peso seco en pitaya. Revista Comalfi 27(2):31-38.

Nascimento, I., Farias, C., Silva, M., Medeiros, J., Espínola Sobrinho, J. y Negreiros, M. 2002. Estimativa da área foliar domeloeiro. Horticultura Brasileira 20(4):555-558.

Palencia, G., Mercado, T. y Combatt, E. 2006. Estudio Agrometeorológico del Departamento de Córdoba. Universidad de Córdoba, Montería, 126p.

Pire, R. y Valenzuela, I. 1995. Estimación del área foliar en Vitis vinifera L. "French Colombard” a partir de mediciones lineales en las hojas. Agronomía Tropical 45(1):143-154.

Queiroga, J., Romano, E., Souza, J. y Miglioranza, E. 2003. Estimativa da área foliar do feijão-vagem (Phaseolus vulgaris L.) por meioda largura máxima do folíolo central. Horticultura Brasileira 21(1):64-68.

Rawlings, J., Pantila, S. y Dickey, D. 2001. Applied Regresión Análisis. Springer, New York, p204-459.

Ribas, F., Cabello, M., Moreno, M., Moreno, A. y López-bellido, L. 2000. Respuesta fisiológica de un cultivo de melón (Cucumis melo L.) a distintas 
dosis de riego. Investigación Agraria Producción y Protección Vegetal 15(3):196-210.

Rossini, A., Deleo, T., Barbosa, J. y Leite, I. 2004. Leaf Area Prediction Models for Zinnia elegans JACQ., Zinnia haageana Regel and 'profusion cherry'. Sci. Agric. (Piracicaba, Braz.), v.61, n.1, p.47-52, Jan./Fev.

SAS. 2007. SAS/STAT guide for personal computers version 9.1 Edition. Cary, NC: SAS INSTITUTE Inc. 378p.

Sepaskhah, A. 1977. Estimation of individual and total leaf areas of safflowers. Agronomy Journal 69(5):783-785.

Squire, G. 1990. The physiology of tropical crop production. C.A.B. International, Wallingford, 236p.

Teruel, D. 1995. Modelagem do índice de área foliar de cana açúcar em diferentes regimes hídricos. Tesis M. Sc. Escola Superior de Agricultura, ESALQ, Piracicaba.

Thornley, J. 1976. Mathematical models in plant and crop physiology. Academic Press, London, 315p.

Walpole, M. 1995. Probabilidad y Estadística. McGraw-Hill, México, D. F., p440443.

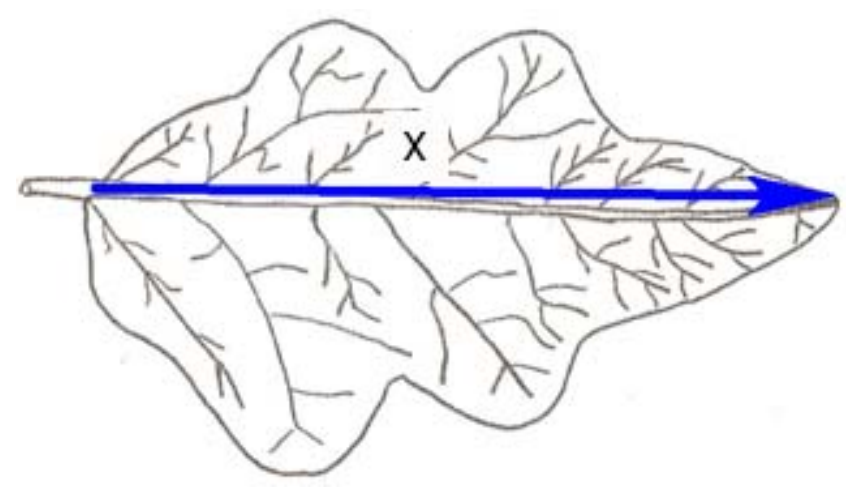

Figura 1. Diagrama de una hoja de berenjena mostrando el largo (X). 
Tabla 1. Análisis de varianza para la regresión del área foliar en berenjena, cultivar "Criollo lila"

\begin{tabular}{cccccc}
\hline Fuente & GL & Suma de cuadrados & $\begin{array}{c}\text { Cuadrados } \\
\text { medios }\end{array}$ & F & P \\
\hline Modelo & 1 & 33960,70 & 33960,70 & 656,67 & $<0,0001$ \\
Error & 27 & 1396,35 & 51,72 & & \\
Total & 28 & 35357,05 & & & \\
\hline
\end{tabular}

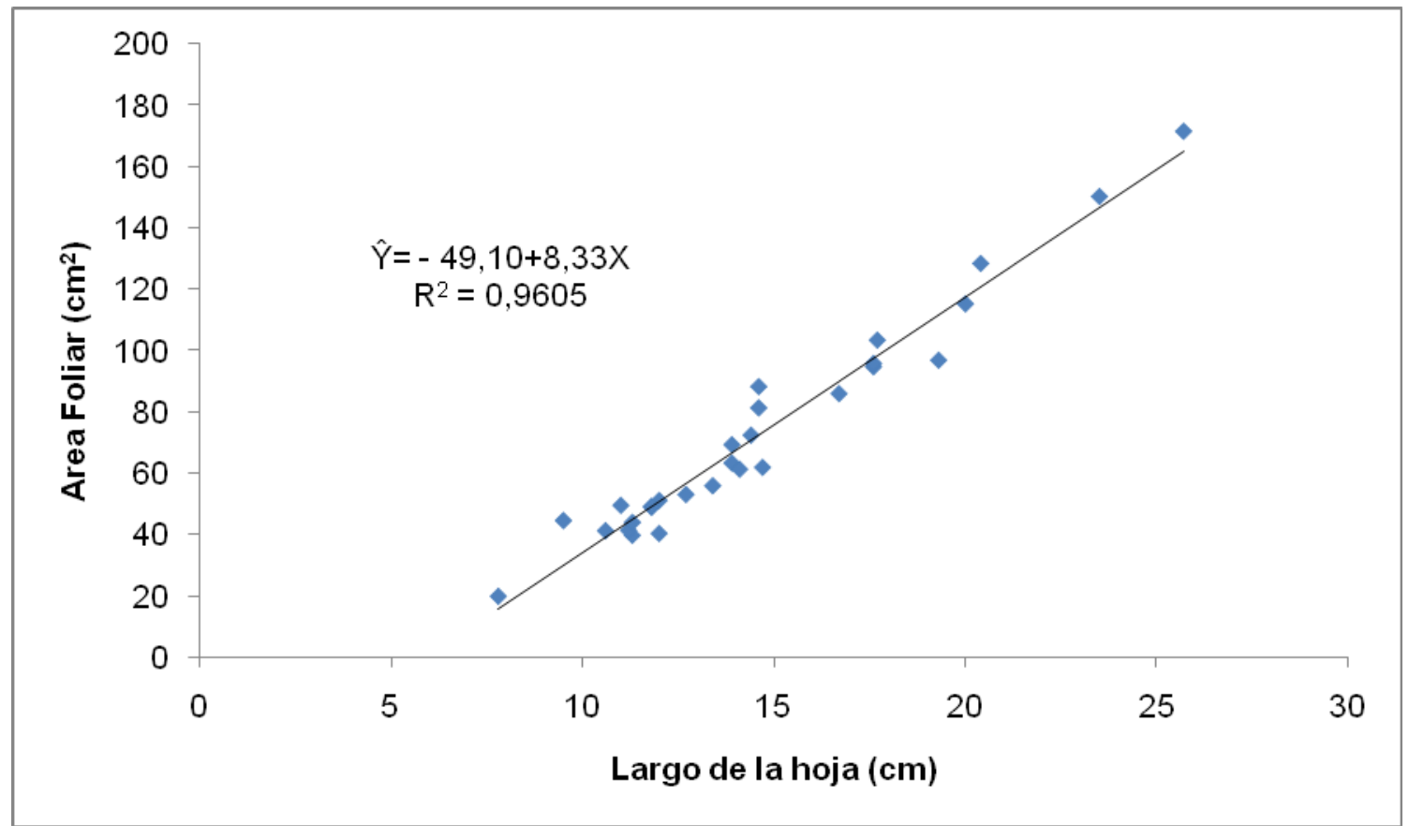

Figura 2. Relación funcional entre el área foliar y el largo de la hoja de berenjena (Solanum melongena L.) cultivar "Criollo lila” 
Tabla 2. Estimación de los parámetros de la regresión del área foliar en berenjena, cultivar "Criolla lila"

\begin{tabular}{ccccc}
\hline Coeficiente & Valor & Error estándar & $\mathbf{T}$ & Pr $>\mathbf{F}$ \\
\hline$\hat{\beta}_{0}$ & $-49,10$ & 4,95 & $-9,92$ & $<0,0001$ \\
$\hat{\beta}_{1}$ & 8,33 & 0,32 & 25,634 & $<0,0001$ \\
\hline
\end{tabular}

